\title{
Der Film als Mittel der Begegnung zwischen den Kulturen
}

\author{
von Ambros Eichenberger
}

Vokabeln und Begriffe wie Weltethos, Weltphilosophie1, Weltliteratur, Weltmusik, Weltkunst usw., wie sie vor allem seit Beginn der 90er Jahre in Umlauf gesetzt worden sind, laden ein, danach zu fragen, ob und in welchem Sinne in Ergänzung dazu auch vor einem "Weltfilm" oder von einer "Weltfilmkultur" gesprochen werden kann.

Als eines der Medien, das breitenwirksam Grenzen überschreitet, scheint der Film auf Anhieb ein besonders gut geeignetes Instrument für diese weltweite, interkulturelle, soziale Kommunikation zu sein, um andere Menschen und Kulturen besser kennen und verstehen zu lernen; und zwar vor allem deshalb, weil das Kino fremde Lebenswelten und Realitäten nicht auf rein verbale und abstrakte Weise vermittelt, sondern diese Realitäten durch bewegte Bilder, Geräusche und Töne anschaulich, vernehmbar und einfühlsam macht.

Im Hinblick auf diese "sinnlichen" und emotionalen Qualitäten des Films sind in den letzten Jahren jene Stimmen denn auch lauter geworden, die dazu aufmuntern, die diesbezüglichen Möglichkeiten des Mediums für den interkulturellen Dialog besser zu nutzen und auszuschöpfen. Viele tun es vor dem Hintergrund der drängenden Probleme unserer Zeit, zu denen Migrationsbewegungen, Fremdenhaß, Rassenkonflikte, neu aufflammende Nationalismen usw. gehören. Sie machen am Ende dieses Jahrtausends ein neues Suchen nach größerer Solidarität und Verständigung zwischen den Völkern und Kulturen unabdingbar. Ganz in diesem Sinn hatte der frühere Generaldirektor der Unesco, Amadou $M^{\prime}$ Bow, ein gebürtiger Senegalese, bereits zu Beginn der 80er Jahre mit eindringlichen Worten darauf hingewiesen, „daß die Menschheit sich zwangsläufig zwischen einem Zeitalter der Solidarität oder einem Rückfall in die Barbarei entscheiden müsse“2.

Solche und andere Statements sind von vielen Medien und

Lic. theol. Ambros Eichenberger OP (geb. 1929) war Filmbeauftragter des "Katholischen Mediendienstes' in Zürich und ist z.Zt. Kulturbeauftragter bei OCIC-Europa in Brüssel.

1 Der Begriff "Weltphilosophie" ist vom jungen Karl Marx schon 1837 im berühmten Brief an seinen Vater, nach der Hinwendung zu Hegel, verwendet worden.

2 Vgl. Dokumentation: Internationale Kulturbeziehungen - „Brücke über Grenzen" (Symposium 80), Baden-Baden 1980, 38. 
Filmemachern, von Kulturpolitikern und einschlägigen Organisationen zum Anlaß genommen worden, den "Auftrag" und die internationalen Kommunikationsmöglichkeiten der Massenmedien, also auch des Films, neu zu überdenken und kritisch zu hinterfragen. So sollte geklärt werden, ob sie die Menschen dümmer, infantiler oder, im Gegenteil, klüger, solidarischer und sicherer machen. Der Hinweis auf die weltweiten (ungelösten) Kommunikationsprobleme signalisiert aber auch ein wachsendes Interesse für eine "Weltkultur", ein Begriff, der sich immer größerer Beliebtheit zu erfreuen scheint. Darin steckt nicht nur die Erkenntnis, sondern auch der Anspruch, eine neue Ära der Kommunikation unter den Kulturen und unter den Völkern in Gang zu setzen. Die Frage, die uns hier beschäftigt heißt, ob und inwiefern der (anspruchsvolle) Film in diesem Annäherungsprozeß die Rolle eines Promotors übernehmen könnte oder sollte. Film also nicht bloß als ein Unterhaltungs-, sondern auch ein Kultur- und ein Kulturvermittlungsphänomen!

\section{Die Zeit der großen Visionen}

Es hat Zeiten gegeben, da waren die Erwartungen in dieser Beziehung sehr hoch, zu hoch gespannt. Sie haben zu Beginn der 80er Jahre zu den großen Entwürfen, Visionen, Leit- und Wunschbildern geführt, die heute mit dem allgemeinen Verlust der Utopien viel von ihrer Anziehungskraft verloren haben. Damals konnten sie sich nicht über mangelnde Popularität beklagen. Vor allem McLuhan's Diktum vom "Global village", das wir seiner Meinung nach den Medien zu verdanken haben, zirkulierte oft recht unkritisch ,durch die ganze Welt'. Spurenelemente davon sind - glücklicherweise - noch immer auszumachen; z.B. im neuen Bericht der UNO-Kommission für "Global Governance" von Shridat Ramphal, der den Titel trägt "Unsere globale Nachbarschaft" ${ }^{\prime 3}$. Von anderen großangelegten Entwürfen, die seinerzeit viel $\mathrm{zu}$ reden, aber auch $\mathrm{zu}$ hoffen gegeben haben, ist heute kaum mehr etwas zu hören. So scheint die Arbeit der „internationalen Kommission zum Studium der Kommunikationsprobleme", die unter dem Vorsitz des Iren Sean Mac Bride von der Unesco 1977 ins Leben gerufen worden war, stillschweigend beerdigt worden $\mathrm{zu}$ sein 4 . Gegen die Medienmacht der Vertreter der "freien" Marktwirtschaft, die ihre materiellen und politischen Interessen durch den Abbau der Ungleichheiten gefährdèt sahen, war nicht anzukommen!

Auch die großen Hoffnungen, die sich mit der von der UNESCO

3 Vgl. Interview mit Shridat Ramphal, Vorsitzender der Kommission für "Global Governance", Tages-Anzeiger (Zürich) vom 1.2.1995, 5.

4 Die Berichte dieser Kommission zum Studium der Kommunikationsprobleme sind 1980 von der deutschen UNESCO-Kommission unter dem Titel „Viele Stimmen - eine Welt" herausgegeben worden. 
angeregten „Weltkonferenz über Kulturpolitik“ (Mexiko, 1982) verbunden hatten, haben sich nicht oder nur sehr sporadisch erfüllt, obwohl die Versammlung von allen Delegierten als "Meilenstein" bezeichnet worden war. In ihrem Mittelpunkt stand das Bekenntnis zur gleichen Würde aller Kulturen. Der Unterschied zwischen "höheren“ und "niederen", wie auch zwischen europäischen und außereuropäischen Kulturen wurde abgelehnt. Es durfte demzufolge nicht mehr herablassend und arrogant von "Subkulturen" die Rede sein. Stattdessen wurde die Aufmerksamkeit auf den "fruchtbaren Dialog" zwischen "freien und gleichen Partnern“ gelenkt, der "beiden Seiten zur Bereicherung diene ${ }^{\prime \prime 5}$. Daß kaum jemand so naiv war, diese Idealvorstellungen mit der Wirklichkeit $\mathrm{zu}$ verwechseln, wurde an der Kritik an den "transnationalen Gesellschaften" ablesbar, die, wie behauptet wurde, "mit ihren Massenmedien und Kulturindustrien die Welt übernommen hätten"6.

Auf der anderen Seite ist aber auch darauf hingewiesen worden, daß die neuen Kommunikations-Technologien nicht nur der Standardisierung der Weltkultur Vorschub leisten, sondern vielen benachteiligten Ländern auch neue Möglichkeiten eröffnen, Kenntnisse über ihre Kulturen, auch über ihre Filmkulturen, zu verbreiten. „Kulturaustausch", so lautete der Tenor, "dürfe niemals eine Einbahnstraße sein, auf der anderen die Kultur derer aufgezwungen werde, die die größere technologische und finanzielle Macht innehätten“7.

Zehn Jahre vorher hatte die katholische Kirche mit der Pastoralinstruktion "Communio et Progressio" (1971) ihre Vorstellungen von den Aufgaben der sozialen Kommunikationsmittel in der modernen Gesellschaft (und in der Kirche) entwickelt und damit ihren Gesprächsbeitrag zum interkulturellen Diskurs geleistet. Mit einem fast ungebrochenen Heils-Optimismus wird dabei die völkerverbindende Funktion der Massenmedien herausgestrichen und mit dem Wunsch oder gar mit der Feststellung verbunden, daß die neuen Technologien "die Zeitgenossen sozusagen um einen runden Tisch" versammeln, so daß sie im "Streben nach Brüderlichkeit (von der Schwesterlichkeit ist noch nicht die Rede) und gemeinsamem Handeln miteinander ins Gespräch“ kommen können (Cep 19).

\section{Merkliche Ernüchterungen}

Im Abstand der seither vergangenen Jahrzehnte hat sich vieles von diesen Zukunftshoffnungen und -modellen aus verschiedenen Gründen

5 Die Berichte zur „Weltkonferenz über Kulturpolitik“ sind als UNESCOKonferenzberichte bei K. G. Saur, München, herausgekommen, vgl. ebd., Nr. 22.39.40.

6 Ebd., Nr. 61, 30.

7 Ebd., Nr. 64, 31. 
als zu optimistisch und als zu theoretisch, vielleicht sogar als "Konstrukt" erwiesen. So werden fremde Kulturen heute doch wohl eher als Bedrohung und weniger als Bereicherung für die eigene Identität empfunden. Und auch der vielbemühte Begriff von der "nationalen Identität" ist im Hinblick auf die wiederaufflammenden Konflikte in Europa und in Afrika dringend einer Revision zu unterziehen. Vor allem aber hat sich herausgestellt, daß den ökonomischen Faktoren zugunsten des kulturellen Diskurses viel zu wenig Rechnung getragen worden ist. Ein Grund dafür liegt vielleicht darin, daß die Bereiche Kultur und Kommerz - nicht nur von den Linken damals wie Feuer und Wasser auseinandergehalten worden sind, während es heute zu Mischformen und zu Annäherungsversuchen kommt, die vielen Sorge bereiten.

Unnütz oder überflüssig waren diese Initiativen und Texte aber deshalb nicht. Schon deshalb nicht, weil sie viele Kreise zum Nachdenken, beispielsweise über die Bedeutung der Kultur, über das Zusammenleben der Kulturen und über die Kulturvermittlung via filmische und elektronische Medien, gezwungen haben. Resultate solchen Nachdenkens sind oder waren auch in politischen Kreisen festzustellen. So hatte der ehemalige Kultusminister eines deutschen Bundeslandes anläßlich eines Symposiums über internationale Kulturbeziehungen 1980 in Bonn festgehalten: "Unser Metier, der internationale Kultur- und Informationsaustausch, ist tatsächlich zur dritten Dimension eines Miteinander in der Weltpolitik herangewachsen. ... Jedes Bemühen, das internationale Gespräch zu stärken, kann heutzutage lebenserhaltend sein"8. Solche Statements fallen ins Gewicht. Die Herrschaftsverhältnisse im Bereich der internationalen Film- und Medienpolitik vermochten sie indessen durch keine neuen, sagen wir Bruder- oder Geschwisterverhältnisse zu ersetzen!

\section{Ungebremste Herrschaft Hollywoods}

Diese Herrschaftsverhältnisse fallen auf dem Gebiet des Kinos und des Films unvergleichlich stärker ins Gewicht als etwa im Bereich der Literatur oder der Musik. Denn in der Filmszene gibt es die universale Dominanz eines bestimmten Typs von Hollywood-Kino, der andere, weniger potente Filmkulturen $\mathrm{zu}$ verdrängen oder gar $\mathrm{zu}$ eliminieren sucht; und dies nicht nur in filmisch unterentwickelten Ländern der sog. Dritten Welt, die auf Importe angewiesen sind, sondern auch in europäischen Gefilden. Das führt so weit, daß beispielsweise in der Schweiz befürchtet wird, der amerikanische Vormarsch könne bald einmal die Leinwandkapazität für eigene und andere hollywoodfremde Produktionen zum Verschwinden bringen. Das Diktum von der Amerikanisierung des Planeten durch das Geschäft mit dem Kino aus

$8 \quad$ Internationale Kulturbeziehungen - Brücke über Grenzen, 14. 
Hollywood ist demnach weit weniger übertrieben als es den Anschein machen kann. Es hat ein "Fundamentum in re", das sich mit Zahlen und Fakten belegen läßt, die hier nicht näher aufgeführt werden können. Es genügt darauf hinzuweisen, daß Actionfilme wie "Speed" und "True Lies", die vor kurzem zu sehen waren, innerhalb von wenigen Wochen im Amerika allein die ,100 Mio. Dollar'-Einnahmengrenze überschritten. Andere Studios haben womöglich noch größere Leistungen vorzuweisen. So sollen die Einnahmen des Disney-Imperiums an "The Lion King" in den ersten Monaten 254 Mio. Dollar betragen haben - mit steigender Tendenz! "Alladin" hat es auf 217 Mio. gebracht. Mit 140 Mio. darf sich auch der Nonnenfilm "Sister Act" noch ganz gut blicken lassen. Wahrlich: das große Publikum, angefangen beim amerikanischen, scheint solche Stories und die Art und Weise, wie sie gemacht sind, mit "unverhohlenem Vergnügen zu goutieren!" Hollywood mit seinen Majors ist im Bereich von Produktion und Distribution unbestreitbare Number one. Die Amerikaner haben ohnehin die Tendenz, nicht nur Lateinamerika, sondern auch die "restliche" Welt als ihren Vorhof zu betrachten. Im Bereich der Movies ist ihnen das ganz offensichtlich besonders gut gelungen. Diese Vorherrschaft der Supermacht mit ihren Superfilmen, deren Qualität sich ja nicht immer bestreiten läßt, wird von zahlreichen Klienten allerdings auch begrüßt; von unzähligen anderen aber als bedrohliche neue Form des Kulturimperialismus em pfunden, der entsprechende antiamerikanische Gefühle weckt. "Sie wollen alles, auch gar alles vermarkten und beherrschen“", meinte neulich ein "Armeleuteregisseur" aus der Dritten Welt - "und sie bewirken dadurch, daß viele kleinere und mittelgroße Verleih- oder Produktionsunternehmen von der Bildfläche verschwinden oder gar nicht erst auftauchen können".

Tatsächlich soll der Kinomarkt noch nie von einer derart großen Anzahl von Kopien amerikanischer Provenienz überschwemmt worden sein wie jetzt. Filme wie "Jurassic Park“ oder der "König der Löwen“ haben dank einer ebenso gigantischen wie genialen Werbestrategie neue Weltrekorde erzielt; und das soll erst der Auftakt für weitere Fortschritte sein, die durch "wunderbare" (sic!) neue Technologien vorbereitet werden. Synthetische Computerbilder, Simulations- und anderen Spezialeffekte werden in Zukunft fast alles möglich und vermarktbar machen, auch das Ausgefallenste und Spektakulärste!

Geographisch ist seit dem Zusammenbruch des Sowjetimperiums für die amerikanischen Movies in ganz Osteuropa ein weiterer großer Markt besetzt und aggressiv erobert worden. Über das Medium Film und die rasanten Entwicklungen im Videobereich kommen die USA dort „, in jede Kneipe, in jedes Kino und in jedes Haus“, wie Kenner der Situation berichten. So stammten bereits 1990 von den zehn neuen Filmen, die im November dieses Jahres in Polen zu sehen waren, acht aus den USA. Ähnlich ist die Situation in allen anderen osteuropäischen Ländern. 


\section{Wachsendes multikulturelles Bewußtsein}

Über die Frage, wie eine zunehmende Expansion des Hollywoodschen Kulturimperialismus gebremst werden könnte, zerbrechen sich nicht nur Japaner, sondern auch Europäer den Kopf. Letztere haben vor kurzem mit den GATT-Verhandlungen kleine Teilerfolge erzielt. Im Interesse eines Plurikulturalismus geht es darum, bewußter zu machen und begründet zu kritisieren, ohne dadurch, das sei abermals unterstrichen, die Qualitäten vieler amerikanischer (Publikums)Filme in Abrede zu stellen, denn das wäre unrealistisch, ungerecht und naiv. Das multikulturelle Bewußtsein, das die Chancenungleichheit zu einem Thema macht, muß zwangsläufig von einem Respekt und von einer Neugierde für andere, nichtwestliche Kulturen getragen werden. Dies sind Qualitäten, die nicht ohne weiteres vorausgesetzt werden dürfen, sondern erst noch zu erwerben sind. Das erfordert viel Toleranz und Verständnis, auch für magische und irrationale Dimensionen und Traditionen, die sich vor den eigenen linear-logischen Denk- und Deutungsmustern unterscheiden. Nur auf diese Weise wird es möglich, daß dieses Andersartige den Beigeschmack des Exotischen, der ihm immer noch anhaftet, (,Sie haben keine Kultur und essen mit den Fingern!") allmählich verliert.

\section{Populäre Publikumsfilme}

Solche Bewußtseinsschübe werden den Siegeszug des amerikanischen Films allerdings kaum aufhalten können. Das Publikum und seine Sehgewohnheiten, die weltweit von amerikanischen Standards geprägt worden sind, leisten ihren Beitrag dazu, auch in Europa! "Wenn das amerikanische Kino die europäischen Märkte zu mehr als 85 Prozent beherrscht, dann deshalb, weil unser Publikum es mit unverhohlenem Vergnügen goutiert", hat Moritz de Hadeln, einer der beiden Leiter der Berliner Filmfestspiele, kommentiert.

Dieses Vergnügen an den Erzeugnissen aus Hollywood, vor allem auch an den Schein-, Konsum- und Gewaltwelten, die sie häufig transportieren, teilt man offensichtlich auch in vielen Ländern der sog. Dritten Welt. Das hat mir neulich ein Regisseur aus Guinea-Bissau mit der Bemerkung bestätigt, daß ein Afrikaner, wenn er zwischen einem afrikanischen oder einem amerikanischen Film wählen könne, sich "todsicher" für den letzteren entscheiden werde. Als Begründung gab er an, „daß sie besser mit dem Publikum funktionieren als unsere eigenen Produktionen"9. Der Hinweis dürfte ins Schwarze treffen. Die Amerikaner machen Publikumsfilme, die auf die seelische Lage der Nation(en) zugeschnitten sind, weil sie diese zuvor testen und bis zur Lancierung eines Films studieren. Wo "es" oder er oder sie (Stars spielen in den

9 Vgl. Flora Gomez (Guinea-Bissau), Paris, „Revue noire“ (1992) Nr. 8, 57. 
USA eine dominierende Rolle und verdienen deshalb astronomische Summen) nicht funktioniert, wird umgebaut, neu komponiert oder sonstwie nachgeholfen. Das gehört zum "business as usual“. Im Unterschied zu Europa wird auf den Autor und seine Rechte (die in der Regel den Studios gehören) keine Rücksicht genommen. Wichtig ist, daß die Einspielergebnisse stimmen. Diesem ökonomischen Denken wird (fast) alles geopfert. Film ist zuerst einmal ein technisch einwandfrei gemachtes Industrieprodukt und eine Unterhaltungsware für die Massen - und dann, je nachdem, vielleicht auch noch Kunst!

\section{Kampf gegen den Kulturimperialismus}

Trotz dieser riesigen Produktions- und Marketingstrategien, die sich außer den Amerikanern niemand auf der Welt leisten kann, auch die Japaner nicht, sollte man die Hollywoodisierung des Planeten nicht wie ein Naturgesetz hinnehmen, auch wenn der Traum von der "einen Welt mit den vielen Stimmen“10 in die Ferne rückt. Abwehrkräfte, die gegen diese kulturelle Überfremdung kämpfen, sind durchaus vorhanden, nicht zuletzt in vielen jungen Filmnationen aller Kontinente, denn dort gibt es eine ganze Reihe von höchst kreativen Autoren, die nicht bereit sind, sich von importierten Fremdbildern bestimmen zu lassen, sondern vielfach - infolge mangelnder Mittel ziemlich zweifelhaft - nach Wegen suchen, um ihre eigenen Realitäten künstlerisch zum Ausdruck zu bringen.

In dieser Beziehung wurden in den letzten Jahren und Jahrzehnten mit anspruchsvollen Filmen doch mancherorts beachtliche Fortschritte erzielt, die auch international von sich reden machten. Das ist mehr als ein vorübergehender Schimmer am Horizont, sondern wohl eher ein Baustein und Beitrag zu den Weltbildern, die das nächste Jahrtausend prägen werden. In Afrika z.B. haben Kulturschaffende, mit oder ohne Hilfe der Regierungen, das Medium Film unmittelbar nach der Entkolonisierung in den frühen 60er Jahren zu einem wichtigen Element im Rückbesinnungsprozeß auf die eigene Kultur und die eigenen Werte gemacht. Ab 1969 hat dann der (schwarz)afrikanische Film in Ouagadougou mit seinem Festival sogar eine "Hauptstadt" bekommen. Sie ist mit dem Ziel ins Leben gerufen worden, "Afrika und der Welt zu zeigen, daß es ein afrikanisches Kino gibt, das in Afrika (also nicht in Paris oder London) gemacht wird, von Afrikanern und für Afrikaner" ${ }^{\prime \prime}$ wie es in den Gründungsunterlagen heißt. Der Kampf gegen die Fremdbestimmung, der in den Anfängen mit dem Slogan "Decolonisez les écrans" recht aggressiv und rhetorisch formuliert und geführt worden ist, greift unter anderem auf die Darstelling von geschichtlichen Episoden dieses "geschichtslosen Kontinents" (Hegel) zurück, um die Bewohner des Kontinents „ihre Würde spüren zu lassen“, wie der

10 Vgl. Bericht der MacBride-Kommission „Viele Stimmen - eine Welt". 
Mauretanier Med Hondo im Zusammenhang mit dem Entstehen seines aufwendigen Filmes "Sarraounia" unterstrichen hat ${ }^{11}$.

Von dieser (afrikanischen) Würde vermögen auch aufgeschlossene Nicht-Afrikaner etwas wahrzunehmen, "weil die bewegten Bilder, die wir produzieren, auch universale Werte enthalten, neben den partikulären Elementen, die für Nicht-Afrikaner aufgeschlüsselt werden müssen, weil sie ihnen nicht ohne weiteres zugänglich sind", hat Flora Gomez, ein bekannter Regisseur aus Guinea-Bissau, seine filmische Philosophie kommentiert ${ }^{12}$. Die Identifikation des Universalen im Partikularen wird der Diskurs zum Thema ,Weltfilm' und ,Weltfilmkultur'stärker als bisher zu beachten und zu analysieren haben.

\section{Das Süd-Süd-Gefälle}

Die Produktionskapazitäten in den Entwicklungsländern, sowohl die materiellen wie die ideellen, sind sehr unterschiedlich. Indien zum Beispiel stellt zweimal soviel Filme her wie die USA und steht damit an der Spitze der ganzen Welt ${ }^{13}$. Der Export dieser Melodramen, vor allem nach Afrika, scheint gut zu funktionieren, und die Akzeptanz beim Publikum ist groß. „In Kuba gibt es praktisch nur Filme aus einem einzigen Land zu sehen, den USA", diese Feststellung eines Kubaners vor der Revolution gilt heute zwar nicht mehr für Kuba, wohl aber für die meisten anderen südamerikanischen Staaten. Daher die immer wiederkehrende Forderung, Platz für nationale Produktionen auf den Leinwänden zu schaffen und die Regierungen zu einer entsprechenden Mithilfe zu bewegen, auf die dieses Anliegen angewiesen ist. Überdies wären dazu entsprechende Distributionsstrukturen erforderlich, die weitgehend fehlen. Das nützt der Konkurrenz aus Hollywood, Indien und Hongkong (Karate und Kung Fu), die ihre B-Pictures ungehemmt verbreiten kann, auch wenn diese, an sozio-kulturellen Maßstäben gemessen, praktisch wertlos sind. Heute erfolgt die Distribution auch über Videotheken, die nicht nur die Städte, sondern bald auch das letzte Dorf erreichen. Das bedeutet keineswegs, daß die Leute die einheimischen Filme nicht sehen wollen, wie vielfach behauptet wird, sondern vor allem auch, daß sie sie nicht sehen können. Dennoch erlebt die internationale Filmszene mit Werken wie "Yeleen" von Souleymane Cissé aus Mali oder "Lebewohl meine Konkubine" von Chen Kaige (Hongkong/Taiwan/China), "Fresa y Chocolate" von Tomas Alea Gutierrez und Juan Carlos Tabio aus Kuba usw. immer wieder kleine Wunder, die nicht produziert worden sind, „um dem weißen Mann (oder der weißen

11 Vgl. Interview mit Med Hondo, Zürich, ZOOM - Zeitschrift für Film, (1987) Nr. 20, 35.

12 Vgl. "Revue noire" (1993) Nr. 8, 57.

13 Die Anteile der Entwicklungskontinente an der Weltfilmproduktion betragen nach dem UNESCO-Programm IPCD für 1984: Afrika 0,4\%, Lateinamerika $6,2 \%$, Asien (ohne China, aber mit Indien) 52,9\%. 
Frau) zu gefallen“, oder um egozentrische Festival-Ambitionen zu befriedigen, sondern um den Reichtum und die Probleme der eigenen Kultur als Teil eines Ganzen ins Ganze einzubringen.

\section{Medien aus der "dritten" in den Medien der "ersten" Welt}

In der "ersten" Welt haben Medien und Filme aus der sog. Dritten Welt, von löblichen Ausnahmen auf Filmfestivals und in Programmnischen einiger Fernsehanstalten wie Channel 4, Arte, oder das ZDF mit dem kleinen Fernsehspiel allerdings immer noch einen recht schweren Stand. Dies gilt vor allem für die Kinos, weil die Verleiher und die Filmtheaterbesitzer sich von "langsamen " und "schwierigen" Filmen aus dem Süden wenig Erfolg an der Kasse versprechen. Immerhin haben besondere Veranstaltungen, teilweise mit bewundernswerter Ausdauer, interkulturelle Vordenker- und Vorreiterdienste geleistet. Im deutschen Sprachraum gehörte die ,Filmwoche Mannheim' zu den Initianten eines Dialogs mit Filmemachern und mit Filmen aus der südlichen Hemisphäre. Dort haben bereits ab 1978 regelmäßig sog. Dritte-Welt-Symposien stattgefunden, die zu vielen fruchtbaren Kontakten und "joint ventures" führten ${ }^{14}$. In Italien ist in Triest mit dem "Festival del Cinema Latino Americano" ein Umschlagplatz für Südamerika entstanden und in Mailand hat die kirchlich orientierte Organisation ,Coe' großen Einsatz für das junge afrikanische Filmschaffen geleistet, u.a. durch die Mitherausgabe der internationalen Zeitschrift "Ecrans d'Afrique”.

\section{Auf der Suche nach mehr Öffentlichkeit}

Diese Möglichkeiten zur Selbstdarstellung von Ländern des Südens im Norden blieben aber meistens auf kleine eingeweihte Kreise beschränkt, die für den Dialog über die eigenen Grenzen hinweg besonders aufgeschlossen waren (und sind). Um ein breiteres (Kino)Publikum für diese Anliegen und Auseinandersetzungen zu erreichen, ist 1986 in der Schweiz mit "Trigon" ein eigener Film verleih für Werke aus der Dritten Welt gegründet worden, der heute auch "Geschäfts"-Verbindungen mit anderen europäischen Ländern sucht und pflegt ${ }^{15}$. Das Unternehmen will seinen Statuten zufolge "qualitätsvolle und völkerverbindende Filme aus der Dritten Welt einem möglichst großen Publikum nahebringen, nicht als kulturell-humanitäres Pflichtprogramm, sondern als bereicherndes Erlebnis, das der Entdeckungsfreude der Zuschauer

14 Eine Dokumentation zum Symposium über den „arabisch-afrikanischen Film" ist von der ,Filmwoche Mannheim' 1979 herausgegeben worden. Vgl. auch die Dokumentationen der folgenden Jahre.

15 Im März 1994 haben sich Teilnehmer aus 10 europäischen Ländern zu einem ersten Verleihsymposium für Filme aus dem Süden in Zürich getroffen. 
entgegenkommt und Zerrbilder korrigiert"16. Das Ziel soll durch neue Bilder und Töne aus (noch) fernen Welten, durch andere Geschichten und Sehweisen, durch noch unbekannte Wertvorstellungen oder solche, die bei uns verdrängt worden sind, durch unverbrauchte Menschenporträts etc. erreicht werden und dadurch dem europäischen Publikum (und eines Tages vielleicht auch dem nordamerikanischen) nützen. Die Kenntnis der andern kann ja auch für das Verständnis der eigenen Identität klärend wirken und damit weit mehr leisten als nur vorhandene Informationsdefizite zu beheben.

\section{Ein humanes Weltethos?}

Es gibt Anzeichen dafür, daß solche Hoffnungen nicht immer aussichtslos bleiben müssen. So hat beispielsweise der südkoreanische Film "Warum Bodhi Dharma in den Orient aufbrach" von Yong-Kyun Bae (1989), ein (Erstlings)Werk, das von der buddhistischen (Zen)Philosophie durchdrungen ist, hierzulande ein großes Publikum anzuziehen vermocht und ganz offensichtlich auf ganzheitliche, mystische Erfahrungen aus Asien - und teilweise auch wieder auf solche aus dem näherliegenden Westen - neugierig gemacht. Mit solchen Erfahrungen werden also nicht nur (spirituelle) Defizite ausgebügelt, um westliche Stagnationserscheinungen durch die Lebendigkeit des Fremden aufzuwärmen oder zu beseitigen. Sie können darüber hinaus auch Suchbewegungen auslösen auf jene humanen Grundbefindlichkeiten hin, die die Menschen, ungeachtet ihrer kulturellen und ethischen Unterschiede, miteinander verbinden. Dadurch wird der Wille zum universellen Verstehen im Sinne einer (Welt-)Ethik der Kommunikation gefördert oder geweckt.

Starke, weil authentische Filme aus China (wie „Dao ma tse" von Tian Zhuang-Zhuang), aus Indien (wie "Piravi" von Karun N. Shaji), aus Lateinamerika (wie "El viaje" von F. E. Solanas), aus dem Iran (wie "und das Leben geht weiter" von Abbas Kiarostami), aus Algerien (wie "Bab-el-Qued City" von Merzak Allouache), aus Burkina Faso (wie "Wend Kuni" von Gaston Kabore) usw., die hierzulande einen Verleiher gefunden haben, können solche Tiefenwirkungen erzeugen und dadurch dem Streben nach Solidarität und Brüderlichkeit Auftrieb geben.

\section{Die Beiträge der katholischen Filmorganisation}

Welchen Beitrag hat die internationale katholische Filmorganisation OCIC, die bereits im Jahre 1928 gegründet worden ist, zur interkulturellen Begegnung mit dem Medium Film geleistet? Eine stillschweigende Einladung dazu ist ja schon durch das Attribut "katholisch" vorgege-

$\overline{16}$ Aus den Zielbestimmungen des „Trigon-Film“-Fördervereins (1988). 
ben, sofern man sich seiner ursprünglichen etymologischen Bedeutung verpflichtet weiß. Bekannte kirchliche Dokumente wie "Communio et Progressio", "Evangelii Nuntiandi", "Aetatis Novae" usw. haben in Anlehnung an die Impulse des II. Vatikanischen Konzils diese völkerverbindende Aufgabe der Massenmedien, also auch des Films, immer wieder in Erinnerung gerufen. Dies gilt nicht zuletzt auch im Hinblick auf eine neue Beurteilung der nichtchristlichen Religionen und den Aufruf, mit ihnen den Dialog aufzunehmen. Dabei ist ausdrücklich auch "von den sozial-kulturellen Werten" die Rede, "die sich bei ihnen finden und die anzuerkennen, zu wahren und zu fördern" sind (vgl. Ad Gentes 7). Wo Filme sich um die Darstellung solcher Werte bemühen, bieten sie ein privilegiertes Feld, um solche Such- und Wahrnehmungsprozesse zu konkretisieren.

\section{Interkulturelle Pioniere}

Dennoch hat sich der Schritt vom westkirchlichen zum weltkirchlichen Kultur-Engagement auch bei der OCIC nicht "automatisch" und ohne Verzögerung vollzogen. $\mathrm{Zu}$ lange, zu einseitig und $\mathrm{zu}$ eurozentrisch blieb der Blick auf die grossen filmischen Manifestationen in Venedig, Cannes und Berlin fixiert. Immerhin darf die Organisation mit ein bißchen Stolz auf die lange Zusammenarbeit mit Pionieren (Frauen und Männern) hinweisen, die für den Filmdialog mit nicht-westlichen Kulturen wichtige Schrittmacherdienste geleistet haben. Dazu gehört z.B. der aus Frankreich stammende Spiritaner-Missionar Jean Vast, der sein ganzes Leben in Afrika (Senegal) verbrachte und dabei auch die Realität und die Möglichkeiten des afrikanischen Films entdeckte und förderte. Nicht in erster Linie durch Vereinnahmung als attraktives Mittel für Pastoral und Katechese im engeren Sinn, sondern selbstwertig, als Ausdruck, Spiegel und Seismograph der Gesellschaft, in der er lebte und lebt. Durch diese Offenheit und Dialogbereitschaft hat sich Jean Vast bei vielen afrikanischen Cineasten große Sympathien erworben, auch bei solchen, die dem Islam oder dem Animismus nahestehen. Die Dienstleistungen, die er mit der Herausgabe einer bescheidenen Filmzeitschrift "UNIR-Cinéma" (lange Zeit die einzige in SchwarzAfrika), mit dem Aufbau einer umfangreichen Film- und Videodokumentation in St. Louis, Senegal, sowie mit der aktiven Teilnahme an vielen filmkulturellen und filmpolitischen Symposien erbrachte, werden heute weit über Afrika hinaus anerkannt ${ }^{17}$.

Ähnliche „Erfolgsmeldungen” gibt es über das Wirken des kanadischen Jesuitenpaters Gaston Roberge in Indien zu berichten. Er war jahrzehntelang in Calcutta, einem der drei großen Zentren der indischen Filmindustrie, tätig und hat vor allem auch die Entwicklung des jungen, engagierten, alternativen, bengalischen Kinos mitverfolgt. Dies tat er so

17 Vgl. „UNIR-Cinéma”, revue du Cinéma africain, Saint-Louis, Sénégal. 
intensiv und kompetent, daß er zu einem persönlichen Freund des großen indischen Meisterregisseurs Satayit Ray geworden ist und auf dessen Wunsch hin die Dialoglisten seiner Filme auf französisch übersetzte. In Lateinamerika hat America Penichet, die vor ein paar Jahren verstorben ist, als Chefin des kontinentalen OCIC-Büros „OCIC$A L$ " dafür gesorgt, daß Südamerika nicht vergessen, sondern in seiner kulturellen Eigenart ernst genommen wurde.

\section{Regionale Schwerpunkte}

Für die Gesamtorganisation, die heute in 130 Ländern ihre Kontaktstellen hat, ist der Filmdialog mit fremden Kulturen 1977 erstmals ein Thema gewesen. Die Studientage des internationalen Kongresses, der damals in München zur Durchführung kam, standen unter dem Leitmotiv "Le cinéma moyen de rencontres entre les cultures" (der Film als Mittel der Begegnung verschiedener Kulturen); sie waren also genau dem Anliegen gewidmet, um das es hier geht. Um den Willen zu diesem kirchlichen und christlichen Engagement im Bereich der internationalen Kulturbeziehungen gleichsam urbi et orbi zu demonstrieren, haben die nachfolgenden OCIC-Kongresse - zusammen mit UNDA - dann systematisch und bewußt in der südlichen Hemisphäre, in Manila, Nairobi, Quito und Bangkok stattgefunden. Ohne den vorausgehenden Aufbau von kontinentalen und regionalen Strukturen, OCIC-Asien, Afrocic, OCIC-AL usw. wäre diese "Auslagerung" nicht möglich gewesen. Sie hat der kirchlichen Filmarbeit in der Folge Türen $\mathrm{zu}$ vielen filmkulturellen Veranstaltungen auch außerhalb der westlichen Welt in Ouagadougou (Burkina Faso), Carthago (Tunesien), Havanna (Cuba), Indien usw. geöffnet. Diese Präsenz vor Ort ist wichtig, um Brücken über kulturelle, konfessionelle und andere Grenzen hinweg zu bauen, zumal sich viele von diesen Veranstaltungen ganz bewußt zu multi-kulturellen Treffpunkten entwickelt haben. Es bestehen somit Gelegenheiten zur universalen Kommunikation - und bisweilen auch zur universalen Konfrontation! -, die in Zukunft wohl über die bisherigen, "gemäßigten" Formen der Interkulturalität hinaus genutzt werden wollen. Denn es ist erwiesen oder es bleibt zu hoffen, $\mathrm{da} ß$ sich dadurch so etwas wie ein Verstehensprozeß über Fragen und Probleme herausbilden kann, die große Teile der Menschheit interessieren und betreffen, weil relevante Filmwerke dazu Anstöße geben. Gestern war das z.B. mit Filmen über oder aus Südafrika der Fall (,Cry freedom" von $R$. Attenborough oder "A World apart" von Chris Menges); und heute sind hier etwa Filmdokumente aus dem ehemaligen Jugoslawien wie "Leben und Sterben in Sarajevo" von Radovan Tadic oder "Tagebuch eines Regisseurs" von Mirza Idrisovic zu nennen.

\section{Initiativen zum Erfolg}

Diese Kontaktmöglichkeiten über viele Grenzen hinweg, haben in den letzten Jahren durch publizistische Initiativen, die der interkulturellen 
Kommunikation förderlich sein wollen, wertvolle Ergänzungen erfahren. So ist die "Collection Cinemedia" über das Filmschaffen in Afrika, die von der OCIC gemeinsam mit dem Verlag L'Harmattan in Paris herausgegeben wird, mittlerweile nicht nur auf 15 stattliche Bände oder Bändchen angewachsen, sondern auch zu einem unentbehrlichen Arbeitsinstrument für all jene geworden, die sich am Diskurs über Afrika und seine filmischen Selbstbilder beteiligen wollen ${ }^{18}$.

Aufs Ganze gesehen sind dies bescheidene Schritte. Aber sie bezeugen den Willen und die Fähigkeit, sich einzumischen, dadurch Zeitgenossenschaft herzustellen und die dialogischen Herausforderungen der Gegenwart und der Zukunft anzunehmen. Die Vielfalt der Aktivitäten erlaubt es leider nicht immer, die Zeit anzuhalten, um den Standort zu überdenken und die Koordinaten für die Zukunft neu zu bestimmen, was angesichts der raschen Entwicklungen notwendig wäre. Denn diese "rasen" auf vielen Ebenen förmlich davon. Sie reichen vom Unbehagen am herkömmlichen, klassischen Kulturbegriff über die Probleme, die sich durch die kulturellen Übergänge heute stellen, bis hin zu den technischen Neuerungen, die den Film durch nichtlineare, digitale und elektronische Bild- und Tonschnitte verändern; von den Auswirkungen der weltweiten Computernetze und Satellitensysteme, die den Planeten erobern, ganz zu schweigen. "Die Welt wird zum Dorf", den Slogan hat neulich ein Kulturkritiker wiederum aufgenommen, um dann gleich hinzuzufügen: "und das Dorf wird zum Dschungel". Solche Entwicklungen sollten nach Möglichkeit verhindert werden. Globales lemen wird demnach in Zukunft noch einen viel zentraleren Stellenwert bekommen (müssen), als es ihn heute schon besitzt. Mit dem Film und seinen Nachfolgemedien!

\section{SUMMARY: Film as a Medium for the Dialogue of Culture}

The main concern of this article is the question in how far the medium ,film' is able to contribute to ,international communication' and, intercultural interaction'. In this context the potential of the culture of a world cinema' is revisited. The visions and the optimistic view of the seventies and eighties are confronted with the disillusions of the presence. Especially, the dominance of the US film business and its cultural imperialism, which now has begun its triumphal march in Eastern European countries, is to be reflected critically. Nevertheless, beside this there are some signs of hope. The author refers to a developing multicultural awareness, which opens up a chance for movies made in 3. world countries'. In this respect, the Catholic film organisations such as OCIC have done some valuable contributions.

18 Vgl. "Collection Cinémedia”, Ed. OCIC/L’Harmattan, Brüssel/Paris. 


\section{RÉSUMÉ: Le film comme moyen de rencontre entre les cultures}

L'article pose la question à savoir dans quelle mesure le média "film" peut contribuer a la communication interculturelle et à la rencontre des cultures. A ce propos, l'auteur s'interroge sur les possibilités d'une culture cinématographique mondiale. L'article rappelle les espérances et les visions des années 70 et 80 et les confrontent avec la situation plutôt désappointante du présent. Il commente surtout de façon critique la dominance mondiale de l'industrie cinématographique américaine et l'impérialisme culturel qui s'y rattache et qui fait à présent son entrée victorieuse dans les pays de l'Europe de l'Est. L'auteur voit pourtant aussi des signes d'espoir et renvoit à un sentiment multiculturel croissant, qui offre aussi une chance aux films des pays du tiers monde. Les activités internationales du travail cinématographique catholique y contribuent de façon précieuse.

\section{RESUMEN: El film como medio de encuentro entre las culturas}

El artícolo se plantea ante todo la pregunta de hasta qué punto el medio "film" puede significar un aporte a la "Comunicación intercultural" y al "encuentro de culturas". El autor se hace la pregunta en este contexto de las posibilidades de una "cultura del film a nivel mundial“. El autor recuerda las esperanzas y visiones de los años 70 y 80 y las confronta con los más bien desencantadores resultados del presente. Comenta de forma crítica principalmente la dominación, extendida a todo el mundo, de la industria del cine Usamericano y el imperialismo cultural que ésto conlleva y que ahora celebra también su marcha triunfal en los países del este europeo. No obstante, el autor vé tambien señales esperanzadoras en la creciente consciencia multicultural que abre posibilidades también a películas del "Tercer Mundo". En este sentido hacen un valioso aporte las actividades internacionales del trabajo filmográfico católico. 